\title{
Newsdesk
}

\section{Mechanism of steroid action}

Steroids are the mainstay of many therapies in ophthalmology and much is known concerning their mode of action particularly at the transcriptional level where they appear to regulate the factor Nfat. However, steroids have multiple effects, many of which are undesirable in terms of their therapeutic antiinflammatory role, and further knowledge of their specific anti-inflammatory role might lead to the development of newer more selective drugs. A recent paper (Nature Medicine 1999;12:1424-7) has shown that glucocorticoids upregulate the expression of a hitherto unknown anti-inflammatory mediator and promote its release from monocytes and macrophages. This factor induces dispersion of leucocytes and lowers their adhesion to endothelial cells. It also inhibits their chemotactic response to fMLP and induces distinctive morphological changes. The activity correlates with a peptide known as thymosin beta4 sulphoxide which is specifically generated by monocytes in the presence of glucocorticoids and acts as a signal to inhibit an inflammatory response. In vivo, the oxidised form of the peptide potently inhibited inflammation in the mouse induced by carrageenin paw. The action of thymosin beta 4 appears to be unique, because oxidation greatly enhances its extracellular signalling properties but appears to downregulate its effects on intracellular G-actin. In fact the authors suggest that this unique mechanism in which a cytosolic protein is converted to an extracellular mediator by methionine oxidation might have implications for drug discovery programmes and the development of new anti-inflammatory strategies.

\section{tPA and intraocular use}

tPA (tissue plasminogen activator) is currently used for a variety of intraocular disorders mostly related to fibrin deposition such as in vitreoretinal surgery for subretinal haemorrhage and for the resolution of fibrin mediated synchiae formation in intraocular inflammation. However, a recent report (Fournal of Cell Science 1999;112:4007-16) has identified a new role for tPA as an activator of microglia. Microglia are the "macrophages of the central nervous system" and have multiple functions, one of which is to participate in CNS inflammation and remove invading pathogens. During this process considerable CNS damage may ensue. Microglia are also the main resident macrophages in the retina and are involved in many inflammatory conditions such as cytomegalovirus (CMV) retinitis, toxoplasmosis, and most posterior segment inflammatory conditions generally. The authors of the new report have shown that tPA may directly activate microglia through a nonproteolytic mechanism - that is, the inactive enzyme is as effective as the active fibrinolytic enzyme. This has implications for ophthalmic surgeons who use tPA to promote clearance of fibrin and blood clots within the eye since high concentrations of the enzyme may activate retinal microglia and induce tissue damage.

\section{IGF-1 is required for VEGF} induced retinal neovascularisation The link between the pituitary and diabetic proliferative retinopathy has been known for many years and indeed formed the basis of early treatments for diabetic retinopathy using pituitary ablation. The nature of the factor released from the pituitary which promoted retinal new vessel formation was not definitively identified and was variously considered to be growth hormone or its downstream mediator insulin-like growth factor (IGF). However, although there were some suggestive in vitro as well as clinical findings that IGF-1 was a possible retinal angiogenic factor, the fact that serum IGF-1 levels were the result of liver synthesis did not agree with the notion that retinal angiogenesis was the result of local retinal ischaemia and the production of growth factors such as vascula endothelial growth by retinal cells. Now Smith et al report that IGF-1 regulates VEGF induced retinal neovascularisation in vivo (Nature Medicine 1999;12:1390-5) in an experimental system using a specific IGF-1 receptor antagonist. The mechanism appears to involve activation of $\mathrm{p} 44 / 42$ mitogen activated protein kinase, establishing a hierar chical relation between IGF-1 and VEGF receptors. The authors suggest that these findings establish an essential role for IGF-1 in angiogenesis and demonstrate a new target for control of retinopathy and may also explain why diabetic retinopathy initially increases with the onset of insulin treatmen since IGF-1 levels rise with newly instituted insulin therapy. In addition, the results indicate why IGF-1 is a risk factor for diabetic retinopathy as has been suggested previously (see Knott RM BFO 1998;82:719-20).

\section{Genomics, proteomics, and now phenomics}

Remarkable advances have been made in the profiling of disease using array technology that allows the detection of many genes on a "chip" - a form of detector which contain many thousands or more of complementary DNA samples that bind mRNA extracts from patient samples and allow the identification of expressed genes or their mutated forms. This represents the advancing discipline of genomics and is itself complemented by proteomics in which genetic products (proteins) are identified on standardised two dimensional gel electrophoresis maps and directly sequenced. The latest development is phenomics which is simply the molecular phenotyping of disease states using a protein chip system that combines the array technology of the genomics system with specific binding technology using surface en hanced laser description/ionisation (SELDI) technology on a protein chip system. The technique allows direct analysis of crude samples and compares samples from patients with normal controls. In a few days or weeks it has been possible to link complex disease states with proteins "in their native biological environments-molecular phenotypes". The methodology has been applied to various forms of cancer and to Alzheimer's disease for the detection of trace amounts of $\beta$ amyloid. Similar technology would be directly applicable to ocular samples such as aqueous humour or vitreous or to extracts of tissues from retinal choroidal biopsies and would allow the detection of, for instance, foreign or abnormal self proteins. These new technologies would appear to have considerable potential for ophthalmic applications.

\section{nvCJD and contact lenses}

The College of Optometrists and the Department of Health, UK, have clashed over a directive that, despite the very low risk of nvCJD (new variant Creutzfeld-Jakob disease), all contact lenses should be restricted to single use wherever practicable. This includes diagnostic lenses and as such has implications for ophthalmologists using contact lenses for ophthalmic examination. The College of Optometrists sees the directive as unworkable and they point out that many pieces of equipment come into direct contact with the eye. Gwyneth Morgan, president of the College of Optometrists said "We are surprised that the Department of Health has taken the stance it has in the light of its continued call for the use of evidence based medicine. We believe that in these circumstances, actions of this nature do not make any sense at all.”

\section{Paclitaxel for chronic progressive multiple sclerosis}

Successful phase I/II trials of micellar paclitaxel, the version of the drug produced by Angiotech Pharmaceuticals (Vancouver, Canada), have recently been reported in a study of 30 patients with chronic progressive multiple sclerosis, a form of the disease which currently has no approved treatment. Paclitaxel is used in several inflammatory conditions including rheumatoid arthritis, psoriasis, and multiple sclerosis. By modifying the drug the company believes it has made it much less allergenic. Its mode of action appears to involve a number of processes including inhibition of leucocyte activation and release of matrix metalloproteinases plus blocking cell proliferation, particularly of endothelial cells at the site of inflammation. In addition, experimental studies have shown that there is a reduction in the proliferation of astrocytes and less gliosis in the neurological lesions.

\section{Chromosome 22 sequenced}

The full sequence of chromosome 22 was published in December last year and marks the first stage in the final push towards the full sequencing of the human genome. An international consortium led by Ian Dunham of the Sanger Centre, Cambridge, UK, and supported by the Wellcome Trust finalised the information and gave a foretaste of what will be available when all 3.5 billion base pairs of the human genome are sequenced. Already the information has proved valuable to researchers such as Professor James Scott of the Department of Molecular Medicine, Hammersmith Hospital, UK. Using a technique of mRNA editing he has mapped the genes of interest to his research in cardiovascular disease to regions of chromosome 22, and he has identified seven new genes at this locus. It will now be possible to study the role of these genes in heart disease at both individual patient and at population level. 